\title{
Proceedings of the 124th Meeting of the Society of British Neurological Surgeons, Royal London Hospital, 21-22 April 1994
}

PROGRESSIVE KYPHOSIS WITH CORD COMPRESSION IN THE RHEUMATOID CERVICAL SPINE

TT King. Department of Neurosurgery, Royal London Hospital, London, UK

In a series of about 160 rheumatoid cervical spines seen over 20 years 70 were treated conservatively, of which five eventually needed operation, and 88 were operated on in a variety of ways. Among the whole group were six cases with a severe and progressive kyphosis affecting the length of the cervical spine from C2-C7. A compensatory lordotic deformity at the craniocervical articulation was present. Cervical cord compression was the indication for operation. The main anatomical and pathological features were involvement of the discs anteriorly and extensive destruction of the apophyseal joint spinous processes, laminae, and ligamentous structures posteriorly. These changes, particularly the posterior ones, allowed toppling of the cervical spine in an anterior direction. The deformity at the atlantoaxial articulation seemed to be compensatory. Surgical treatment has proved difficult. Posterior stabilisation is unsatisfactory. Limited success has been obtained by the use of an anterior fibula graft combined with cancellous bone. This seems more likely to succeed than multiple interbody fusions.

STEREOTAXIC TARGET SELECTION FOR THALAMOTOMY FOR MOVEMENT DISORDERS OCE Sparrow, ES Watkins. Wessex Neurological Centre, Southampton and Royal London Hospital, London UK

Stereotaxic thalamotomy is a regularly, if infrequently performed procedure for movement disorders such as Parkinson's disease, dystonia musculorum deformans, intention tremor from trauma and multiple sclerosis, and benign essential tremor. Although a resurgence of interest in the use of posteroventral pallidotomy, as first practised by Leksell, has been reported by Laitinen et al, ${ }^{1}$ various targets, related to various fixed points has been used. These are based on the foramen of Monro, the anterior commissure, and the posterior commissure. Some surgeons relate target coordinates to the foramen of Monro, some to the anterior commissure, and some to the midpoint of the intercommissural line. Laitinen ${ }^{2}$ obtained their preferred radiological target from 14 different stereotaxic surgeons, related to the midpoint of the intercommissural line. With this map, the favoured target after stimulation for physiological localisation was demonstrated in a series of over 100 thalamotomies.
1 Laitinen LV, Bergenheim AT, Hariz MI. Leksell's posteroventral pallidotomy in the treatment of Parkinson's disease. Neurosurg 1992;76:53-61.

2 Laitinen LV. Brain targets in surgery for Parkinson's disease. Results of a survey of Parkinson's disease. Results of a survey of
neurosurgeons. $f$ Neurosurg 1985;62: 349-51.

INVESTIGATION OF POSTOPERATIVE RECURRENT SCIATICA

BD White, GFG Findlay. Walton Centre for Neurology and Neurosurgery, Liverpool and Department of Neurosurgery, University Hospital, Nottingham, UK

During recent years the radiological investigation of recurrent sciatica after lumbar discectomy has evolved from myelography through enhanced CT to the current gold standard of multiplanar, multiple sequence, gadolinium enhanced MRI.

To define the minimum imaging required to confidently distinguish between recurrent disc and epidural scarring, a blind retrospective analysis was undertaken with 20 such cases investigated since January 1991. Multimodality analysis showed that symptomatic disc recurrence was characterised by an anterior or anterolaterally placed extradural mass at the level of the disc space or pedicle, occupying more than one third of the spinal canal width and elevating the dural tube. In contrast, postoperative scarring was confined to the level of the disc space, extended laterally around the canal drawing the dural tube towards it and when anteriorly placed occupied less than one quarter of the canal width. Assessing the contribution of each sequence to the final diagnosis, precontrast T1 weighted or proton density scans were sufficient to enable confident clinical management of the patient. Additional T2 weighted and gadolinium enhanced $\mathrm{T} 1$ weighted scans allowed more precise radiological distinction of scar from disc but did not alter eventual management.

If the morphology of symptomatic postoperative extradural masses are taken into consideration, single sequence MRI is sufficient in most cases to differentiate recurrent lumbar disc herniation from epidural scarring. Multiple time and money consuming investigations may be reserved for those few cases where doubt remains.

REPORT OF A NATIONAL NEUROSURGICAL TELERADIOLOGY SYSTEM BASED ON OPTICAL SCANNING TECHNOLOGY

WP Gray, TF Buckley. Wessex Neurological Centre, Southampton, UK and Department of Neurosurgery, Cork Regional Hospital, Ireland

In a previous report to this society, it was shown that optical scanning technology was a promising technique for capturing high resolution CT images for teleradiological transmission. We have further developed this technology and have designed and installed a national neurosurgical teleradiology system in Ireland based on optical scanning.

With Telematique grant funding, dedicated $x$ ray scanners were developed to digitise hard copies of CT and MRI scans. These scanners use charge coupled devices to convert the light transmitted through the radiographs to an analogue voltage, which is then digitised. This digital data is read into a transmitting computer that transfers the electronic image to the remote computer in the receiving neurosurgical unit. Here the image can be viewed and manipulated. The system is PC based running under Microsoft Windows. Data transmission is over leased data lines at $128 \mathrm{~Kb}$ per second.

The system has been installed in four remote sites draining into the two neurosurgical centres in Ireland. The system is in full clinical use with over 400 emergency CT scans successfully transmitted to date. The system is simple to use, has gained widespread acceptance, and delivers an image quality superior to many commercially available systems.

EMERGENCY MEDICAL IMAGE TRANSMISSION BY FAX

G Solanki, C Marks, R Macfarlane. Departments of Neurosurgery, Royal London Hospital, London and Cork Regional Hospital, Cork, Eire

The benefits of image transfer are well known to neurosurgeons and radiologists. Unfortunately, cost is a major constraint, particularly if systems are to be made widely available at reasonable cost or indeed in developing countries.

A novel method is described for emergency medical image transmission, which relies largely on available technology. The technique involves the reproduction of a CT, MRI, or $x$ ray film with a new direct print photographic paper that produces an image of photographic quality in $90 \mathrm{sec}$ onds. This is then transmitted by a 64 grey scale fax machine.

To evaluate the efficacy and image quality of this system, a consecutive series of 10 scans sent to the neurosurgery unit at the Royal London Hospital from peripheral units for urgent neurosurgical opinion, were then faxed to neurosurgery registrars and consultant neuroradiologists at five neurosurgery centres. Their opinions were then compared with that of a neurosurgeon and a neuroradiologist who viewed the original image. Examples of the image quality and results were discussed. 
ELECTRICAL MAPPING OF THE SPINAL CORD JC Sutcliffe, TH Koeze. Department of Neurosurgery, Royal London Hospital, London, UK

The dermatomal pattern of sensory innervation of the skin has been established for many years, but despite this, methods of stimulation of the spinal cord produce inconsistent results in terms of localisation of the evoked responses experienced by the patients.

An attempt has been made to map out the areas associated with stimulation of the spinal cord, during neurostimulation procedures for pain, to determine those areas most closely associated with the sensory innervation of "difficult" regions to treat, such as the lumbar spine and the perineum.

Stimulation at D1 produced evoked responses over the chest and aborted the pain of angina (also improving left ventricular function). Stimulation at D8 most consistently produced evoked responses in the lower back. The perineum (bladder, vagina, and rectum) was mostly affected by stimulation at $\mathrm{D} 9 / 10$. As the electrode moved caudally at this region, the evoked responses moved cranially and "deeper" into the perineum.

Chronic pain in the lower back and perineum are notoriously difficult to treat with neurostimulation, but inserting the electrodes under local anaesthesia and identifying the evoked responses in the appropriate region with the starting points indicated gives the best chance of success.

CEREBRAL RESPONSES TO TRIGEMINAL NEURALGIA PAIN MEASURED BY POSITRON EMISSION TOMOGRAPHY (PET)

ND Kitchen, H Watabe, AKP Jones, DGT Thomas. Department of Neurological Surgery, Institute of Neurology and MRC Cyclotron Unit, Hammersmith Hospital, London, UK

Although trigeminal neuralgia is clinically well defined little is known about the central representation of the pain experience.

Five patients with right sided trigeminal neuralgia each underwent two PET studies with the opiate analogue ligand ${ }^{11} \mathrm{C}$ diprenorphine; the first before RF-thermocoagulation, the second after successful treatment (mean visual analogue score changing from 45 ("in pain") to 0 ("completely pain free")). Tegretol doses were unchanged between scans. For each subject the two PET studies were coregistered and subtracted on a pixel to pixel basis to obtain precisely the focal brain changes in ${ }^{11} \mathrm{C}$ diprenorphine binding. These changes were then averaged across the five subjects.

Findings were consistent in all patients with global increases in binding after treatment. Significant focal increases $(p<0.01)$ were found in the prefrontal and anterior cingulate cortices and thalamus. Interestingly, these changes were bilateral but more prominent on the right and did not correlate with changes in regional cerebral blood flow, which were widespread and non-specific. Increased binding of ${ }^{11} \mathrm{C}$ diprenorphine when pain-free suggests that endogenous opiates are no longer filling receptor sites and indicates that the opiate modulated medial pain system is important in the central response to trigeminal neuralgia pain.
NEUROSTIMULATION FOR PAIN RELIEF: THE ROYAL LONDON HOSPITAL EXPERIENCE JK Harris, JC Sutcliffe, TH Koeze, $S$ Mooney. Department of Neurosurgery, Royal London Hospital, London, UK

Neurostimulation is an accepted treatment for the relief of chronic pain. The equipment is expensive and the treatment requires a dedicated, specialised follow up and maintenance service. The results of neurostimulation for pain are reported in $\mathbf{8 0}$ patients who required 90 stimulator systems.

The mean duration of symptoms before neurostimulation was 11.5 (range 1-31) years, with a mean follow up period of $4 \cdot 1$ (range $0 \cdot 3-14 \cdot 5$ ) years. Sixty seven per cent of patients reported to clinicians that they experienced at least $50 \%$ relief of their pain, $19 \%$ reported no relief. Twenty one patients experienced complete or near complete resolution of their pain at some time during treatment. The outcome reported to clinicians was compared to that documented by a disinterested third party investigator. Complications of the procedure included a wound infection rate of $5.5 \%$ and surgical repositioning of the electrode in $17 \%$. An assessment was made of the results according to clinical diagnosis and stimulation type, site, and operative variables.

THE SHEFFIELD EXPERIENCE IN THE TREATMENT OF ACOUSTIC NEUROMA BY STEREOTACTIC RADIOSURGERY: THE LEARNING CURVE

DMC Forster, AA Kemeny, P May, PS Dias. Royal Hallamshire Hospital, Sheffield, UK

Fifty five acoustic neuromas have been treated by radiosurgery in Sheffield. Of these 29 tumours in 27 patients have been followed up for more than four years. Most patients had central neurofibromatosis, with some considered unsuitable for microsurgery and some who refused to consider it. Tumours were bilateral in 18 and unilateral in nine. Initially the radiation dose chosen was similar to that used for AVMs and the growth of 11 out of 17 tumours was controlled (seven decreased in size) but five patients developed facial weakness and three a sensory deficit and only three of the nine with useful hearing were able to maintain it afterwards. Therefore the dose to the periphery of the lesion was reduced from 2500 to $1750 \mathrm{cGy}$. In the subsequent 12 tumours 11 have been controlled (six decreased) with one facial weakness and one sensory loss with preservation of hearing in four out of the six with useful hearing. Four of the seven failures were over $3 \mathrm{~cm}$ in diameter. Not surprisingly smaller tumours can be treated better and more safely. The reasons for failure were discussed. With careful case selection and radiation dosage, influenced by the need to preserve hearing, radiosurgery makes a valuable alternative to microsurgery.

INTRACRANIAL REPAIR OF THE FACIAL NERVE WITH FREEZE-THAWED SKELETAL MUSCLE GRAFT

MG O'Sullivan, IR Whittle, M Glasby. Departments of Neurosurgery and Anatomy, University of Edinburgh, Edinburgh, UK
Facial nerve injury occurs not infrequently during surgery in the cerebellopontine angle (CPA). A novel method of facial nerve repair in the CPA with freeze-thawed skeletal muscle as a nerve graft is reported.

A posterior fossa craniectomy was performed in six adult sheep. The facial nerve was divided in the CPA and a freeze-thawed skeletal muscle interposition graft $^{1}$ was inserted and secured with fibrin glue. After nine months graft function was assessed by electrophysiological and anatomical methods. Successful graft function was obtained in all animals.

Hence, freeze-thawed skeletal muscle autografts are a convenient method of repairing the facial nerve in the CPA in sheep.

1 Glasby MA. Nerve growth in matrices of orientated muscle basement membrane: developing a new method of nerve repair. Clinical Anatomy 1990;3:161-82.

VISUAL OUTCOME AFTER TRANSPHENOIDAL SURGERY IN ELDERIY PATIENTS

DNP Thompson, P Kelland, J Acheson, $M$ Powell. Departments of Surgical Neurology and Neurophthalomology, National Hospital for Neurology and Neurosurgery, London, UK

Pituitary adenoma is uncommon in elderly patients. Overt endocrinological symptomatology is rare and the diagnosis is often only apparent once symptoms of mass effect supervene. Visual outcome after transphenoidal hypophysectomy in 22 elderly patients is reported.

Patients of 65 years and over who had undergone transphenoidal hypohysectomy for pituitary tumours with suprasellar extension were reviewed retrospectively. Clinical records, preoperative imaging, and preoperative and postoperative ophthalmic assessments (including Goldmann perimetry) were examined in all cases. A technique of instillation of saline into the CSF via a lumbar catheter was used in most cases to aid tumour descent and facilitate removal.

Twenty two patients aged 65 and over (range 67-83 years) were identified. In all cases visual impairment was the primary presenting feature. The duration of symptoms varied from two weeks to 20 years. CT and/or MRI confirmed suprasellar extension in all cases.

A visual field defect (of one quadrant or more) was present in 20 patients. After surgery 14 improved (seven of these to normal), seven were unchanged, and one patient was worse. Visual acuity was reduced in 18 cases at presentation. An improvement to $6 / 12$ or better after surgery was seen in 11 cases (four to normal), five were unchanged, and two were worse. The degree of ophthalamic deficit was a better predictor of outcome than the duration of symptoms. There was no mortality. Complications comprised CSF leak (two cases), pneumonia (one case), and transient diabetes insipidus (two cases). Six patients required long term endocrine replacement therapy. The most common pathological diagnosis was chromophobe adenoma (17 cases).

In conclusion, transphenoidal hypophysectomy may safely afford significant improvement in visual function in elderly patients with suprasellar pituitary adenoma. Neither age nor duration of symptoms 
should preclude surgical intervention in this group of patients, whose independence would be severely threatened by visual failure.

USE OF NEUROSURGICAL INTENSIVE CARE AFTER ELECTIVE INTRACRANIAI

PROCEDURES: IS THERE A SAFE PERIOD? NWM Thomas, WAS Taylor, JA Wellings, BA Bell. Atkinson Morley's Hospital, London, UK

A postoperative haematoma can occur after any intracranial procedure and is one reason for intensive nursing observation in the early postoperative period. In the United Kingdom this is usually done in an intensive care unit for an empirical period of time but normally overnight. This can cause pressure on the availability of intensive care unit beds and this study aims to establish whether there is a "safe period" after which patients can be discharged to a ward for further observation.

All of the elective intracranial procedures excluding shunts at Atkinson Morley's Hospital over a five year period have been retrospectively reviewed. Between 1988 and 1992, 1993 elective intracranial operations were carried out comprising 17 burr hole biopsies, 288 stereotactic biopsies, 1427 elective craniotomies, and 261 elective posterior fossa explorations. Postoperative haematomas that required reoperation occured in 39 patients $(1.9 \%)$. No patients having a burr hole biopsy and one patient $(0.3 \%)$ having a stereotactic biopsy had a haematoma evacuated. This patient deteriorated within two hours of the procedure. In the patients having elective craniotomies, deterioration occured during two time intervals: within six hours of the procedure in 24 patients, and later than 24 hours after the procedure in five patients. Of the posterior fossa explorations, patients who deteriorated did so within four hours of the procedure.

It is concluded that patients who have had elective craniotomies, burr hole biopsies, and stereotactic biopsies, and have recovered to their preoperative state by six hours, can be discharged to a ward. Sudden deterioration associated with hydrocephalus after posterior fossa procedures would still warrant overnight observation in an intensive care unit.

INTRACRANIAL PRESSURE MONITORING IN THE MANAGEMENT OF HYDROCEPHALUS AND SHUNT RELATED PROBLEMS

J Fouyas, RD Hayward, ATH Casey, WFJ Harkness. Department of Neurosurgery, Hospital for Sick Children, London, UK

Although the clinical and radiological diagnosis of hydrocephalus is often straightforward, there is a minority of cases in which the decision whether to shunt or not can be extremely difficult. The diagnosis of a blocked shunt system is usually made without difficulty, but there are some patients who present with atypical symptoms and signs that may be due to non-valve related conditions and others in whom headache and vomiting are caused by overdrainage rather than blockage of the shunt.
A retrospective study was carried out of complex cases of hydrocephalus in whom 24 hour intracranial pressure (ICP) monitoring with the Camino system was used either as an aid to initial diagnosis or as part of the investigation of complex symptoms occurring in previously shunted patients.

Thirty seven patients are reported, 17 of whom were being assessed for the presence of ICP sufficiently high to justify the insertion of a shunt system. Of these eigh showed a significant increase in ICP whereas the pressures were normal in nine. The remaining 20 patients already had a shun system in situ and were being investigated for symptoms that could have been due to intermittent shunt blockage, over drainage of CSF, or conditions unrelated to either hydrocephalus or the shunt system. Of these, 14 proved to have overdrainage as demonstrated by a dramatic fall in ICP when the patient was moved from the horizontal to the vertical position. There was no significant morbidity associated with the procedure.

In conclusion clinical and radiological criteria alone may afford insufficient information in both the initial evaluation of hydrocephalus and the subsequent management of the shunted child. ICP monitoring has been a safe means of investigating such cases and has provided valuable information on which to base surgical management.

TESTING EXPLANTED SHUNT VALVES

REASONS FOR VALVE FAILURE

HL Brydon, RD Hayward, WFJ Harkness, R Bayston. Hospital for Sick Children, London and City Hospital, Nottingham, UK

Most shunt valves that are removed are discarded with at the most bacteriological examination, but improvements in valve design will only occur if failed valves are examined and the cause of failure determined.

Over a 15 month period, 43 explanted valves were submitted to a four part study: $65 \%$ of them were Hakim valves, which is the standard valve for the unit. The test procedure comprised flow pressure testing opening and closing pressure determination, measurement of the tendency to syphon, and finally dismantling and inspection of the internal structure of the valve. Overall, $77 \%$ of valves failed to meet the manufacturers' specified performance data, even though the preoperative and perioperative cause of shunt failure was thought to lie outside the valve. Over $84 \%$ of valves with metallic parts had accumulated debris internally, and this was thought to have impaired their performance. By contrast only $25 \%$ of non-metallic valves contained internal debris, a significant finding $(p<$ 0.01 ) that contradicts the published claim that metal parts would cut away debris. ${ }^{1} \mathrm{All}$ of the valves had a high tendency to overdrainage.

Attention is drawn to the high number of malfunctioning valves and it is recommended that a change of valve should be considered in all shunt revisions.

1 Hakim S, de la Roche FD, Burton JD. A critical analysis of valve shunts used in the treatment of hydrocephalus. Dev Med Child Neurol 1973;15:230-55.
LONG TERM PROGNOSIS FOR CHILDREN WITH TRETED HYDROCEPHALUS: A 10 YEAR FOLLOW UP

ATH Casey, E Kimmings, A Kleinlugtebeld, WAS Taylor, WFJ Harkness, RD Hayward. Hospital for Sick Children, London, UK

The short term complication rate of shunt insertion in hydrocephalic children is widely reported: however, there is a paucity of information on their long term future.

The aims of this study were to determine the surgical morbidity, mortality, and long term intellectual and functional outcome of a cohort of shunted hydrocephalic patients.

The case records of a cohort of 155 shunted hydrocephalic patients from 1978-83 were analysed thus ensuring a 10 year follow up period. Criteria for admission to the study included a hospital record describing aetiology, treatment, complications, and final outcome at 10 years. Statistical analysis was performed by $\chi^{2}$ tests with $\mathrm{p}<0.05$ regarded as significant.

One hundred and fifty five patients (90 male, 65 female), with an age range of 0.1-164 months (median $4 \cdot 1$ months) at the time of first shunt insertion, were analysed. Aetiologies were recorded as congenital $32 \%$, intraventricular haemorrhage $21 \%$, aqueduct stenosis $14 \%$, infection $12 \%$, neoplasm $11 \%$, and other miscellaneous causes; $53 \%$ of patients attend normal school and $36 \%$ require specialist schooling; $19 \%$ are severely handicapped; and $44 \%$ of patients have required no revisions. A total of 225 revisions ( 1.45 per patient) were performed over the 10 year period. These were mainly attributable to blockage $49 \%$, infection $19 \%$, or disconnection $13 \%$. The 30 day infection rate was $5 \cdot 2 \%$ for first insertion and $4.9 \%$ for revisions; $92 \%$ of revisions for infection occurred within three months of insertion. The risk of blockage was more than three times greater for revisional surgery compared with first insertions (NS). Those children requiring multiple revisions did not have a poorer intellectual outcome, nor did the aetiology of hydrocephalus affect outcome.

This study provides much needed long term prognostic information for counselling of parents whose children are about to undergo shunt placement.

AUDIT OF THE MEDOS PROGRAMMABLE

SHUNT: A PRELIMINARY ANALYSIS

UK Shunt Audit Group. Academic Neurosurgery Unit, Addenbrooke's Hospital, Cambridge, UK

A nationwide audit of MEDOS programmable valves placed in 138 patients (mean age 44.8 , range two days to 84 years) thought to be at higher risk of complications from shunt surgery, has been conducted to evaluate the complication rate in MEDOS shunted patients in addition to clinical outcome and resource consumption (Smethwick, Cambridge, Maudsley, Southampton, Wakefield, Belfast, Sheffield, Royal London, Newcastle, St Bartholomew's, Coventry, Plymouth and Dublin contributed patients).

Of the 149 valves inserted, 22 were removed: 18 due to infection (overall rate $12 \cdot 1 \%$ ) and four due to shunt malfunction. 
There were 13 shunt revisions. Programming failure occurred in four shunts. On average there were 1.7 (range 1-9) setting changes overall with 0.9 (range $1-6)$ in the first postoperative month; $33 \%$ of shunts had no setting change after insertion. Subdural collections occurred in 24 $(16 \cdot 1 \%)$ patients after shunting. On average, patients underwent 4.6 (range 1-25) CTs and 5.7 (range 1-20) check radiographic investigations postoperatively. The mean duration of inpatient stay was 25.6 days; for infected cases 46 days, and for non-infected cases 22 days. Twenty two patients were discharged to residential care.

IS NEUROSURGICAL FOLLOW UP ON HYDROCEPHALIC CHILDREN VALUABLE?: A PROSPECTIVE STUDY

E Kimmings, A Kleinlugtebeld, ATH Casey, J Fouyas, WFJ Harkness, RD Hayward. Hospital for Sick Children, London, UK

Seventy newly diagnosed hydrocephalic patients undergo a shunting procedure each year at Great Ormond Street with an additional 1.4 shunt revisions being required over a 10 year period. Current unit policy is to re-evaluate these children in the neurosurgery clinic on an annual basis. This places a considerable burden on limited medical resources, however, and potentially compromises the efficient management of other outpatients. The value of routine clinics is debatable, and a prospective study was therefore carried out to investigate this matter. Exclusion criteria from the study were tumour associated hydrocephalus, a shunt procedure within the previous six months, or a consultation requested by a patient The following patient characteristics were obtained: age, number of operations, and the presence of physical or mental handicap.

Three hundred and twenty five patients were seen over a six week period in the neurosurgical outpatient department. Eighty five patients representing $26 \%$ of the total number of neurosurgery patients had an appointment for routine hydrocephalus follow up. Twenty three children $(27 \%)$ failed to attend compared with $15 \%$ of the remaining neurosurgical patients. Of the 62 attenders, one family declined interview. The mean age was 7.5 years (range 8 months to 17 years). Sixty six per cent of patient's families stated that they had specific questions that they wished to raise with their doctor. Sixty one patients had previously received written information abou hydrocephalus that they had found valuable $(97 \%)$. Thirty nine per cent would be content for either general practitioner or local hospital follow up. On the other hand the neurosurgeon considered that at least $46 \%$ of patients did not seem to be seen on a regular basis-the two groups were not concordant. In $21 \%$ of consultations a new problem was discussed and in eight patients $(13 \%)$ this led to a change in management.

It is concluded that routine outpatient review is not required in a neurosurgical setting and that follow up can equally well be performed with local facilities with a caveat that there are good lines of communication to deal with shunt problems.
COMPUTERISED LUMBAR INFUSION TEST: MULTICENTRE EXPERIENCE IN CLINICAL STUDIES IN HYDROCEPHALUS

M Czosnyka, F Gjerris, W Maksmowicz, $M$ Roszkowski, H Whitehouse, E Guazzo, PJ Kirkpatrick, P Smielewski, JD Pickard. Academic Neurosurgical Unit, Addenbrooke's Hospital, Cambridge, Department of Neurosurgery, University Hospital, Copenhagen, Denmark, and Warsaw Medical Academy and Children's Health Centre, Warsaw, Poland

The computerised lumbar infusion test was designed to compensate for the disadvantages of Katzman's lumbar infusion method: poor accuracy of estimation of the resistance to CSF outflow (rCSF) and poor predictive value in NPH. Accuracy is improved by computer intracerebral pressure (ICP) signal processing and model analysis for measurement of cerebrospinal compensatory variables including rCSF, brain compliance, pressure-volume index, estimated sagittal sinus pressure, CSF formation rate and other variables. The computerised test has been used for a five year multicentre study in 350 hydrocephalic patients of various ages, aetiology, and states of cerebrospinal compensation:

The test measures rCSF as accurately as the reference lumboventricular perfusion study (correlation $r=0.98 ; \mathrm{n}=32$ ).

The results provide an indication of poor outcome after shunting if the major variables are in the normal range-that is, rCSF less than $13 \mathrm{~mm} \mathrm{Hg} / \mathrm{ml} / \mathrm{min}$, PVI above $13 \mathrm{ml}$, and resting ICP below $15 \mathrm{~mm} \mathrm{Hg}$.

In ventricular dilatation in children the test is helpful to differentiate between CSF circulation disorders of various origins.

The test permits reliable assessment of shunt malfunction: a functioning shunt shows a specific pattern dependent on shunt type. The computerised test is a reliable method for the assessment of disturbances of the CSF circulation.

ANATOMY OF NEUROVASCULAR COMPRESSION OF THE LOWER CRANIAL NERVES

PJ Hamlyn. St Bartholomew's Hospital, London, UK

For trigeminal neuralgia and hemifacial spasm alone probably more than 50000 neurovascular decompression operations have been performed worldwide. The incidence with which significant vessels have been found, however, has varied dramatically between authors in both anatomical work on previously unaffected cadavers as well as in the reports of operative findings in afflicted patients. A new method of in vitro perfusion has been established allowing accurate delineation of the anatomy in control cadavers and it demonstrated a lower incidence of compressive vessels than in a matched population of patients with trigeminal neuralgia. ${ }^{1}$ The significance of this has been tested in the present study.

Meta-analysis of the literature shows significantly higher incidence of neuralgia on the right side, in females rather than males and with increasing age. Hemifacial spasm is more common in females but does not affect one side more than another and has a less linear relation with age.

The present work contains the results of 137 dissections of control cadavers. The epidemiology of trigeminal neuralgia and to a lesser degree hemifacial spasm, was followed by the proximity of vessels to nerves. The statistical significance of these results and their implication was discussed.

1 Hamlyn PJ, King TTJ. Neurosurgery 1992;76: 948-54.

OUTCOME OF SURGERY FOR CHRONIC

TEMPORAL LOBE EPILEPSY: ANTERIOR TEMPORAL LOBECTOMY $v$ SELECTIVE AMYGDALOHIPPOCAMPECTOMY

SP Harland, IA Robertson, CE Polkey. The Neurosurgical Unit, Maudsley Hospital, London, UK

One hundred and nineteen patients underwent surgical resection for chronic drug resistant temporal lobe epilepsy at The Maudsley Hospital between 1987 and 1992. Anterior temporal lobectomy (ATL) was performed on 80 patients (mean age $27 \cdot 0$ (range $3 \cdot 6-57$ ) years; mean age of onset 10.0 (range 0 to $39 \cdot 2$ ) years). Selective amygdalohippocampectomy (AHE) was performed on 39 patients (mean age 29.0 (range 9.9 to 48.9 ) years; mean age of onset 10.8 (range 0 to 40.9 ) years). All patients were investigated preoperatively with ECG, CT and/or MRI scanning, and psychometry. All operations were performed by one of the authors (CEP) with intraoperative electrocorticography (ECoG). Anterior temporal lobectomy consisted of a 5-6 cm en bloc resection. Selective AHE was carried out as described by Yasargil et al (1985). Follow up ATL was mean $2 \cdot 6$ (1-5), AHE mean 3.7 (1-5). Anterior temporal lobectomy produced significantly more patients who were seizure free (Engel Grade 1; ATL 53.8\%; AHE 41\%). This may be attributed to the greater number of tumours in this group (ATL 38.9\%; AHE $5 \cdot 2 \%)$. The main determinant of outcome (Engel grade) in both groups was pathological diagnosis (table): tumours (DNET, glioma, hamartoma) were associated with a good prognosis as was medial temporal sclerosis; Non-specific changes were associated with a poor prognosis. In this series there was no significant change in psychometry postoperatively and thus the suggested neuropsychological benefit of AHE was not seen.

\begin{tabular}{|c|c|c|c|c|}
\hline \multirow[t]{2}{*}{ Outcome } & \multicolumn{4}{|c|}{ Anterior temporal lobectomy (\%) } \\
\hline & Tumour & $C D$ & MTS & NS \\
\hline \multirow{4}{*}{$\begin{array}{l}\text { No/rare seizures } \\
\text { Worthwhile } \\
\text { improvement } \\
\text { No improvement }\end{array}$} & $77 \cdot 4$ & $66 \cdot 6$ & $67 \cdot 8$ & 20 \\
\hline & $\begin{array}{r}16 \cdot 1 \\
6 \cdot 5\end{array}$ & $\begin{array}{l}16 \cdot 7 \\
16 \cdot 7\end{array}$ & $\begin{array}{l}21 \cdot 4 \\
10 \cdot 7\end{array}$ & $\begin{array}{l}33 \cdot 3 \\
40\end{array}$ \\
\hline & \multicolumn{4}{|c|}{$\begin{array}{l}\text { Amygdalohippocampectomy } \\
(\%)\end{array}$} \\
\hline & Tumour & & & NS \\
\hline \multirow{2}{*}{$\begin{array}{l}\text { No/rare seizures } \\
\text { Worthwhile } \\
\text { improvement } \\
\text { No improvement }\end{array}$} & 100 & & & 25 \\
\hline & $\begin{array}{l}0 \\
0\end{array}$ & & & $\begin{array}{l}62.5 \\
12.5\end{array}$ \\
\hline
\end{tabular}

$\mathrm{CD}=$ Cortical dyspalsia; MTS $=$ medial temporal sclerosis; NS = non-specific

IS WADA TESTING HELPFUL IN LATERALISING SEIZURE FOCUS BEFORE TEMPORAL LOBE SURGERY FOR EPILEPSY?

CL Mallucci, AR Walsh, EH Rolfe. Departments of Neurosurgery and Neuroradiology, Queen Elizabeth Medical Centre, Birmingham, UK 
Wada testing has been used routinely for 30 years to lateralise hemisphere dominance, and to identify: people at risk of a severe amnesic syndrome after temporal lobe surgery for epilepsy. Using the Montreal memory function assessment, which tests recall recognition of items presented for memorisation during the test, it's use has been evaluated in lateralising seizure focus compared with neurophysiological methods. Patients whose memory function performed equally well bilaterally or whose dominant hemisphere performed better on testing were lateralised as having a non-dominant focus, and those with better function in the non-dominant hemisphere as having a dominant hemisphere focus.

Twenty three Wada tests have been evaluated in this unit. Overall, Montreal testing correctly lateralised the focus in 18 cases (sensitivity $78 \%$ ), and incorrectly lateralised in five $(22 \%)$. There was no significant difference between dominant and non-dominant foci. A good postoperative outcome (Engel grades 1 and 2) was achieved in 74\% overall and this was not significantly affected by concurrence or non-occurrence of Wada and neurophysiological testing. It is concluded that preoperative Wada memory testing provides useful confirmatory evidence of seizure focus lateralisation.

EVERYDAY SPATIAL MEMORY AND ORIENTATION AFTER SURGERY FOR TEMPORAL LOBE EPILEPSY

EA Maguire, ET Burke, JP Phillips, H Staunton. Richmond Institute for Neurology and Neurosurgery, Beaumont Hospital, Dublin, Eire

The role of temporal neocortex and mesial temporal lobe structures in humans in the formation and storage of internal spatial representations of an everyday real life environment was examined in 20 patients who had undergone unilateral temporal lobectomy or neocorticectomy (11 left, nine right) for the relief of intractable epilepsy. Patients and 10 matched controls were exposed to video presentations of routes through novel urban area. Learning and memory were then assessed across several variables.

Analyses showed deficits after both left and right temporal lobe surgery. Both patient groups required significantly more exposures to the video before a criterion level of learning was reached $(p<0.01)$, both were impaired on tests of route planning and execution $(p<0.001)$, and in sketch map representations of the stimulus area $(p<0.001)$. Analysis of error types in recognition memory during learning, however, showed that the left temporal group made significantly more omission errors ( $<0.01$ ), whereas the right temporal group made significantly more inclusion errors $(\mathrm{p}<0.05)$.

Furthermore, patients with right temporal lesions were also significantly impaired relative to controls and the left temporal group, on tests of landmark selection ( $p<$ $0.01)$, proximity $(\mathrm{p}<0.05)$, and distance $(p<0.01)$ judgements.

Findings argue for a role of both left and right temporal lobe structures in different aspects of spatial representation.
FUNCTIONAL ASPECTS OF CALLOSAL SURGERY

AT King, CE Polkey. Neurosurgical Unit, Maudsley Hospital, London, UK

Callosal section is an empirical treatment for epilepsy that can appreciably relieve atonic (drop) attacks and episodes of status epilepticus. Twenty four partial callosal sections are reviewed, including seven patients with gross unilateral hemisphere disease. Preoperative carotid amytal tests have been used to assess bilateral secondary synchrony and intraoperative electrocorticography to indicate the effectiveness of the callosal section during the procedure. The benefit of each of these techniques has been evaluated.

The 24 patients comprised 14 males and 10 females; eight of the 24 patients were aged 15 or less. There was follow up of more than one year for 22 patients. Overall 12 of these 22 patients gained benefit in terms of fit reduction from the surgery. Three of the patients with unilateral hemisphere disease benefitted. The other four patients subsequently underwent major resection. Eighteen patients had preoperative amytal investigation. In eight of these the test suggested that one hemisphere was driving the other. Four of these patients obtained fit reduction including two with unilateral hemisphere disease.

Intraoperative electrocorticography was attempted on 23 patients, but useful data were obtained in only 13 patients. Among these 13 there was a good correlation between ECoG desynchronisation and eventual outcome in eight patients.

DYSEMBRYOPLASTIC NEUROEPITHELIAL

TUMOURS

ATH Casey, WFJ Harkness, A Raymond, H Cross, RD Hayward, B Harding. Departments of Neurosurgery and Neurology, Hospital for Sick Children, London, UK

Present classification of brain tumours, such as that of the World Health Organisation are inadequate for the characterisation of certain paediatric tumours. Recognising this problem Daumas-Duport retrospectively analysed a group of complex neuroepithelial tumours from her institution and identified a group of 20 patients with uniform pathological and clinical features. She coined the term dysembryoplastic neuroepithelial tumour to describe this abnormality.

We have critically reviewed the clinical neurophysiological, psychological, patholog ical, and radiological studies in a series of 10 patients ( 6 male; 4 female), mean age 5.2 years (seven months to 11 years) in an attempt to further characterise this recently described condition. All patients presented with medically refractory epilepsy, complex (eight) or simple partial seizures (two) with generalisation of seizures in two patients. One patient had headache and a temporal bone swelling. The EEG was abnormal in all the cases reviewed with localised slow activity and interictal spiking. The EEG abnormalities were more extensive in three patients than the observed lesion. Bitemporal changes were seen in two patients

Magnetic resonance imaging was the investigation of choice although the lesion was visualised on CT. The lesion was predominantly intercortical with occasional involvement of the the temporal lobe typically in close proximity to mesial temporal structures. Features of MRI included circumscribed hyperintensity on long TE/TR images, hypointensity on short TR images, and cyst formation.

Surgical follow up ranged from eight to 46 (mean 19.7) months. Nine patients are currently seizure free (Engel I). One patient is experiencing a reduced number of seizures (Engel III). There were no serious surgical complications.

Identification of this group of tumours is advantageous as it spares these patients the deleterious effects of radiotherapy, chemotherapy, and unnecessarily aggressive surgery.

LIPOPROTEIN (a) CONCENTRATIONS IN CEREBROVASCULAR SACCULAR ANEURYSMAL DISEASE

C Bolger, D Croke, P Collins, M Farrel, JP Phillips, D Bouchier-Hayes. Departments of Neurosurgery, Biochemistry, and Vascular surgery, Beaumont Hospital, Dublin, Ireland

Lipoprotein (a) $(\mathrm{Lp}(\mathrm{a}))$ is a serum lipoprotein. Raised serum concentrations of $\mathrm{Lp}(\mathrm{a})$ are a known independent risk factor for ath erosclerosis. Serum $\mathrm{Lp}(\mathrm{a})$ concentration are strongly genetically determined Cerebrovascular aneurysmal disease (CAD) is a common condition, but its aetiology remains controversial. It remains unclear to what extent, if any, CAD and atherosclerosis are related. Serum $\mathrm{Lp}(\mathrm{a})$ concentrations have been measured in 50 patients with angiographically established saccular aneurysmal disease. These were compared with a group of age and sex matched normal healthy controls. Mean age (SD) of cases was $50(13.9)$ years and $46(12.9)$ years for controls. Cases and controls were free of clinically significant atheromatous disease as judged by history, examination, ECG, and carotid angiography in the cases.

The mean serum $\mathrm{Lp}(\mathrm{a})$ concentration in cases was 32.9 (SD 29.6) $\mu \mathrm{g} / \mathrm{dl}$ compared with $17 \cdot 8(19 \cdot 2) \mu \mathrm{g} / \mathrm{dl}$. This corresponds to $\log$ normalised values of $2.3(1 \cdot 1)$ for controls and $3.0(1 \cdot 1)$ for cases $(t=3.015, \mathrm{p}<$ $0.003)$. If only subjects younger than 50 years were considered the difference remained significant $(t=2.351, p<0.02$, $\mathrm{n}=21$; controls, $\mathrm{n}=24$ cases). These results indicate that either cerebrovascular aneurysmal disease and subclinical atherosclerosis are related, as evidenced by raised serum $\operatorname{Lp}(a)$ concentrations or that $\operatorname{Lp}(a)$ is a risk factor for vasculopathies other than atheroma.

COMPARISON OF THE EFFECT ON SURVIVAL OF SURGICAL RESECTION OF A SINGLE BRAIN METASTASIS FROM LUNG $v$ NON-LUNG PRIMARY TUMOURS

JAL Miller, D Collie, P Rothwell, R Grant. Department of Clinical Neurosciences, Western General Hospital, Edinburgh, UK

Surgical excision followed by radiation therapy (RT) has been advocated as the 


\begin{tabular}{|c|c|c|c|c|c|c|c|c|}
\hline & \multirow[b]{2}{*}{$\begin{array}{l}\text { Age }(y) \\
\text { Median } \\
\text { (range) }\end{array}$} & \multirow{2}{*}{$\begin{array}{l}\text { Karmofsky } \\
\text { score } \\
\text { Median } \\
\text { (range) }\end{array}$} & \multirow[b]{2}{*}{$\begin{array}{l}\text { Supra- } \\
\text { tentorial } \\
(\%)\end{array}$} & \multirow[b]{2}{*}{$\begin{array}{l}\text { Infra- } \\
\text { tentorial } \\
(\%)\end{array}$} & \multicolumn{3}{|c|}{ Treatment (\%) } & \multirow{2}{*}{$\begin{array}{l}\text { Survival } \\
\text { (days) } \\
\text { Median } \\
\text { (range) }\end{array}$} \\
\hline & & & & & $\begin{array}{l}R T \\
(\%)\end{array}$ & $\begin{array}{l}C_{x} \\
(\%)\end{array}$ & $\begin{array}{l}R T+C_{X} \\
(\%)\end{array}$ & \\
\hline \multicolumn{9}{|l|}{ Lung: } \\
\hline $\begin{array}{l}S \pm M(n=6) \\
M(n=23)\end{array}$ & $\begin{array}{l}63(61-67) \\
64(51-81)\end{array}$ & $\begin{array}{l}75(30-90) \\
70(40-90)\end{array}$ & $\begin{array}{l}67 \\
70\end{array}$ & $\begin{array}{l}33 \\
30\end{array}$ & $\begin{array}{l}50 \\
91\end{array}$ & $\begin{array}{l}0 \\
0\end{array}$ & $\begin{array}{l}33 \\
9\end{array}$ & $\begin{array}{l}144(50-225) \\
125(32-337)\end{array}$ \\
\hline \multicolumn{9}{|l|}{ Non-lung: } \\
\hline $\begin{array}{l}S \pm M(n=6) \\
M(n=15)\end{array}$ & $\begin{array}{l}62(40-74) \\
58(27-69)\end{array}$ & $\begin{array}{l}70(60-90) \\
80(60-90)\end{array}$ & $\begin{array}{l}75 \\
60\end{array}$ & $\begin{array}{l}25 \\
40\end{array}$ & $\begin{array}{l}67 \\
62\end{array}$ & $\begin{array}{l}0 \\
8\end{array}$ & $\begin{array}{c}17 \\
31\end{array}$ & $\begin{array}{l}422(33-723) \\
115(17-508)\end{array}$ \\
\hline
\end{tabular}

predict the efficacy of mannitol therapy, pretreatment data was categorised by either treatment success or failure where success is defined as treatment that either reduces ICP below 20 or increases CPP above $60 \mathrm{~mm} \mathrm{Hg}$. With the exception of CPP pulse amplitude $(p=0.0074)$, which reflects the lower pretreatment CPP found in the treatment success group, there were no significant differences between treatment success/failure data for any pretreatment ICP waveform indices. When waveform

treatment of choice in patients with an accessible single brain metastasis and a good performance score. We assessed the survival from onset of neurological symptoms in adult patients with lung or nonlung primary tumours treated by either combined surgical and medical treatment (RT and/or chemotherapy $\left(C_{x}\right)$ ) or medical treatment alone.

Data was obtained from retrospective analysis of the South East of Scotland Brain Tumour Register for incident cases during 1989/90.

A single metastasis was present in 54 patients with primary lung tumour and 33 with non-lung primary tumours (breast $(30.5 \%)$, bowel $(30.5 \%)$, and others $(39 \%))$. Only 29 patients with lung tumour and 21 patients with non-lung tumours were suitable for active treatment. The median survivals were similar for small cell lung tumours and adeno/squamous cell lung tumours. The results are presented in the table.

In conclusion the role for excision of single metastasis from lung tumours should be reassessed.

AUDITING OUTCOME IN NEUROSURGICAL

PATIENTS

KS O'Neill, JA Wellings, BA Bell. Atkinson

Morley's Hospital, London, UK

Audit is a growing and important aspect of clinical practice and measuring outcome is an essential part of that process. To achieve this a seven point audit scale (AMH score) was devised to measure disability and outcome in neurosurgical patients. This was shown to be reliable when tested for observer variation and sensitivity. ${ }^{1}$ Our detailed computer database the input of which is the discharge summary allows collection of admission and discharge AMH and Glasgow coma scores on all patients. Additional input has been follow up AMH scores obtained by outpatient or telephone interview at three months, to assess outcome. An internal control of 50 patients showed no significant difference between scoring the same patients by outpatient or telephone review. From 2323 neurosurgical admissions between June 1992 and May 1993 , there were 131 patients who were lost to follow up (6\%). There were 238 different diagnostic Read codes and 13 patients had no diagnostic code recorded $(0 \cdot 6 \%)$. The codes were divided into eight diagnostic categories: tumours (26\%), degenerative conditions $(25 \%)$, vascular disease $(20 \%)$, trauma $(13 \%)$, infection (4\%), congenital lesions $(3 \%)$, neurological conditions $(7 \%)$, and miscellaneous diagnoses (2\%). The mean three month outcome AMH scores for the eight groups were $4,5,4,5,5,6,6$, and 5 respectively, showing that most patients were independent and most were able to work. Further analysis of the vascular group, which had a large variability in outcome, illustrates the range of information the follow up system provides. The worst diagnostic subgroup were spinal infarcts, the best were AVMs, with mean AMH scores 4 and 6 respectively. Subarachnoid haemorrhage occured in 180 patients with a mean outcome score of 4 ( $\pm 2 \mathrm{SD})$. Of the $25 \%$ patients who had died at three months, their average Glasgow coma score on admission was seven. The table shows the changes in $\mathrm{AMH}$ score between admission and discharge and three month assessment, revealing the improvement in good outcomes between discharge and follow up, and the death of all the patients discharged vegetative (score of 2 ) by three months.

\begin{tabular}{lrrrrlrc}
\hline \multicolumn{1}{c}{$A M H$ Score (\%) } \\
\cline { 2 - 7 } (Dead) & 1 & \multicolumn{1}{c}{2} & \multicolumn{1}{l}{3} & \multicolumn{1}{c}{4} & \multicolumn{1}{l}{5} & 6 & 7 (Noma) \\
\hline Admission & 0 & 22 & 35 & 13 & 17 & 6 & 7 \\
Discharge & 16 & 4 & 29 & 15 & 16 & 11 & 9 \\
3 months & 25 & 0 & 9 & 9 & 19 & 19 & 19 \\
\hline
\end{tabular}
1 Watkins LD, Bell BA, Marsh HT, Uttley D. Neurosurg 1990;4:403-5.

INDICES OF ICP WAVEFORM SHAPE CORRELATE WITH CEREBRAL PERFUSION PRESSURE BUT FAIL TO PREDICT EFFICACY OF MANNITOL THERAPY FOR RAISED ICP IR Piper, S Midgley, NM Dearden, FC Wong, MJ Souter, ID Miller. Department of Clinical Neurosciences, Western General Hospital, Edinburgh, UK

It is known that the intracranial pressure (ICP) waveform shape changes with cerebral perfusion pressure (CPP), although it is less clear which components of the waveform correlate with CPP and whether these changes provide useful information for managing patients with raised ICP.

In a prospective study carried out over two years, ICP, blood pressure and CPP waveform data were collected from 20 head injured patients using on line minute by minute derivation of 12 indices of the ICP waveform shape. Physiological data including heart rate, core temperature, arterial and jugular venous oxygen saturation $\left(\mathrm{SaO}_{2}\right.$, $\mathrm{SjvO}_{2}$ ), and end tidal $\mathrm{CO}_{2}$ were also collected.

Data from 40 mannitol treatments were obtained from 12 patients (eight focal and four diffuse brain injuries). There was no significant difference in physiological or ICP waveform variables when either the pretreatment or post-treatment data were classified by type of head injury (focal/diffuse) or by presence of hyperaemia. To determine if ICP waveform variables can indices pretreatment and post-treatment were compared four variables changed significantly ( $p<0.01$ ): ICP pulse amplitude, ICP distortion factor $k$, the pulsatility index of the CPP, and the amplitude transfer function (XFRaLF) of the fundamenta harmonic. No waveform indices based solely on high frequency components were found to change significantly with CPP Further analysis of those waveform variables found to change significantly with mannitol is continuing for other forms of treatment particularly those likely to have a more uniform "vascular" effect.

DETERMINATION OF CEREBRAL PERFUSION PRESSURE THRESHOLDS IN HEAD INJURED PATIENTS

FC Wong, IR Piper, JD Miller. Departmen of Clinical Neurosciences, Western General Hospital, Edinburgh, UK

The management of cerebral perfusion pressure (CPP) in head injured patients requires invasive monitoring of blood pressure, intracranial pressure (ICP), and other physiological variables. Transcranial Doppler (TCD) monitoring can also be useful in the management of such patients. The main aim of this paper was to determine the CPP thresholds in individual patients below which treatment might be required.

Head injured patients who were ventilated in the intensive care unit were studied and had multiple physiological variables monitored. Simultaneous samples of blood pressure, ICP, and TCD signals were obtained and their derived variables from waveform analysis displayed on a personal computer in real time.

Ten patients were studied during spontaneous and treatment induced periods of changing CPP. A total of 16 recording episodes were carried out and this generated about 2700 data points. With a sequential analysis of variance, a breakpoint near $70 \mathrm{~mm} \mathrm{Hg}$ of CPP was shown for the grouped data when the pulsatility index of the middle cerebral artery waveform was plotted against CPP. There was good correlation between the pulsatility index and the fundamental transfer function (from blood pressure to ICP) data in 11 out of the 16 recording episodes, when plotted against CPP.

The CPP breakpoint values could, however, differ by at least $10 \mathrm{~mm} \mathrm{Hg}$ between and within patients on different days.

In conclusion, an on line computerised monitoring system of blood pressure, ICP, and TCD waveforms can help to determine the CPP threshold values, which could differ between individual patients. This breakpoint value may be determined by other variables besides the pulsatility index of the middle cerebral artery Doppler waveform. 
THE EARLY MANAGEMENT AND USE OF THE INJURY SEVERTTY SCORE IN SEVERE HEAD INJURY

RS Cooke, BP McNicholl, DP Byrnes. Royal Victoria Hospital, Belfast, UK

A 12 month prospective study of the early management of severe head injury (SHI) in Northern Ireland was performed. Glasgow coma scale (GCS) and injury severity score (ISS) criteria were used to define SHI. GCS, ISS, and TRISS scores were related to outcome at one year, with the rank correlation coefficient.

The Glasgow coma score defined 78 patients, and ISS a further 53 as having SHI $(n=131), 27 \%$ were hypoxic and $18 \%$ were hypotensive on admission to the primary hospital. Endotracheal intubation was performed in $92 \%$ of comatose patients, and adequate resuscitation was performed in $87 \%$ of those transferred; $61 \%$ were moved to the neurosurgical unit (with a mean time until transfer of over three hours); $60 \%$ were comatose, $68 \%$ were intubated; and $74 \%$ had an anaesthetic escort. Thirty eight patients (29\%) required evacuation of an acute intracranial haematoma; $68 \%$ of operated patients had not been classified as SHI by GCS. The overall mortality was $38 \%$.

Injury severity score identified patients with severe intracranial pathology who required neurosurgical intervention and with poor outcomes that the GCS based definition of SHI did not. TRISS and GCS correlated similarly with one year outcome, based on both Glasgow outcome score and mortality.

It is concluded that the early management of SHI is reasonable with high rates of intubation, but that there are problems with prehospital oxygenation and transfer. We suggest that ISS and TRISS could be used in local audit, and for inter-regional and historical comparison of head injury management.

DEPRESSED SKULL FRACTURES:

CONSERVATTVE OR SURGICAL MANAGEMENT? KS O'Neill, WAS Taylor, JA Wellings, BA Bell. Atkinson Morley's Hospital, London, UK

There is continuing debate on the correct management of depressed skull fractures, in part due to the variation in severity of injury. It is usually recommended that depressed fragments are raised to restore normal anatomy and allow debridement of damaged tissues. We reviewed 120 patients with depressed fractures admitted between 1986 and 1993. Details of mode and severity of injury were assessed and outcome with an average follow up of 23.8 months measured in terms of complication rate and the Glasgow outcome score. There were 96 $(80 \%)$ compound fractures and $24(20 \%)$ closed fractures. Forty eight patients $(40 \%)$ were treated conservatively and $72(60 \%)$ were treated with surgical elevation and debridement. Most compound fractures with underlying brain injury were treated surgically $(84 \%)$. Of the 63 compound fractures without brain injury, $32(51 \%)$ were treated with surgical elevation and 31
(49\%) conservatively. There was no significant difference in outcome scores between the two groups, $96 \%$ of both having a good outcome. The surgical group, however, had four patients $(12 \cdot 5 \%)$ with complications, epilepsy (one), focal deficit (one), and cosmetic deformity (two). The conservative group had only one complication $(3 \cdot 2 \%)$, a patient with a cosmetic deformity and the groups were otherwise comparable. Average depths of depression were not significantly different between the groups, but all the patients with complications had depressions $>10 \mathrm{~mm}$ in depth. We conclude that some patients with no underlying brain injury can be safely managed by conservative wound toilet and closure, without bony fragment elevation, which may be associated with increased morbidity and be an unnecessary intervention.

COMBINED SINGLE STAGE REPAIR IN CRANIOFACIAL TRAUMA

J Martin, M Danford, DA Lang, BTE Evans, G Neil-Dwyer. Wessex Neurological Centre and Maxillo-facial Department, Southampton General Hospital, Southampton, UK

Management of craniofacial trauma has traditionally been neurosurgically led. The definitive repair was delayed because of concern that early surgery would incur an increased morbidity and mortality. In the United States there has been a trend towards early single stage repair of craniofacial trauma. From August 1988 until December 1993, 59 patients with craniofacial trauma were admitted to the Wessex Neurological Centre. In this retrospective analysis the aim was to determine the effects of earlier surgery not only on morbidity and mortality but also on function and cosmesis.

Of the 59 patients 55 were male and the mean age was 27 years. Twelve were unsuitable for repair due to the severity of their injuries. These patients subsequently died. The remaining 47 underwent combined craniofacial repair. There were no deaths in this group. Ten $(21 \%)$ were operated on within 48 hours of injury, $10(21 \%)$ between 48 hours and one week, 11 (23\%) between eight and 14 days, five (11\%) between 15 and 21 days, $10(21 \%)$ between 21 and 54 days, and one patient at 11 months. At six months 11 were classified as severely disabled whilst the remaining 36 were either moderately disabled or had made a good recovery. The outcomes were as expected from consideration of the Glasgow coma score after resuscitation.

Early reconstructive surgery is technically easier. This approach has been adopted at many centres in the United States, ${ }^{1}$ but not in the United Kingdom. With careful patient selection, we have shown that early craniofacial repair is not associated with any increased morbidity or mortality, and produces satisfactory functional and cosmetic results. The changing role of neurosurgery in the management of craniofacial trauma was emphasised as well as the importance of a multidisciplinary team in the patient selection process.

1 Benzil D, Ribotti E, Dagi T, et al. Neurosurgery 1992;30:166-71.
CLINICOPATHOLOGICAL OBSERVATIONS IN 100 CONSECUTIVE FATAL HEAD INJURIES ADMITTED TO A NEUROSURGICAL UNIT

DP O'Brien, M Bennett, JP Phillips, MA

Farrell. Departments of Neurosurgery and Neuropathology, Beaumont Hospital, Dublin, Eire

Trauma is the leading cause of death between 20 and 40 years of age and cerebral injuries account for most trauma related deaths. A retrospective clinical and neuropathological study of 100 consecutive fatal head injuries admitted to a neurosurgical unit was carried out to determine the incidence of primary diffuse axonal injury (DAI) and of secondary hypoxic-ischaemic encephalopathy (HIE). The clinical, necropsy, and coroner's records of 100 fatal head injury cases admitted to the neurosurgical unit were examined. The mode and location of injury, interhospital transfer, contributing factors, and hospital course were recorded. All cases had a full neuropathological assessment including brain maps, photographs, and histological slides. Standard pathological criteria for the assessment of DAI and HIE were used. A full systemic necropsy was performed in each case. The male to female ratio was 3:1 (age range 2-88, mean 40 years). Accidents occurred most commonly on the streets $(66 \%)$ and at home $(21 \%)$. Motor vehicle accidents $(53 \%)$ and falls $(36 \%)$ accounted for most injuries. Alcohol consumption was the most frequent contributory factor in adults (31\%). Neuropathological examinations showed focal mass lesions in $68 \%$ of cases, acute subdural haematomata being the commonest (45/68; 66\%). Neuropathological evidence of $\mathrm{HIE}, \mathrm{DAI}$, and raised intracranial pressure were recorded in $74 \%$, $42 \%$, and $89 \%$ respectively. Nineteen patients showed evidence of both DAI and HIE. Fifty two per cent of cases had significant extracranial injuries of whom it was considered that seven died from these injuries.

The results from this study show a high incidence of potentially avoidable cerebral damage (HIE) in fatal head injuries. Although a time lapse between certification of brain death and discontinuation of life support systems may have contributed to the high incidence of HIE, the results still indicate scope for improvement in terms of the prevention of secondary cerebral injury and also indicate a need to increase awareness of potentially life threatening extracerebral injuries as a cause of coma.

ASSESSMENT OF CEREBRAL HYPOXIA USING NEAR INFRARED SPECTROSCOPY DURING CAROTID ENDARTERECTOMY

PJ Kirkpatrick, P Smielewski, PC Whitfield, M Czosnyka, JD Pickard. University Department of Neurosurgery, Addenbrooke's Hospital, Cambridge, UK

Near infrared spectroscopy (Hamamatsu NIRS 1000) was used to monitor the oxygenation state of the brain during carotid endarterectomy in 13 patients. During surgery, a continuous measure of oxygenated haemoglobin $\left(\mathrm{HbO}_{2}\right)$, deoxygenated haemoglobin $(\mathrm{Hb})$, oxygenated cytochrome $\left(\mathrm{CytO}_{2}\right)$, and the total haemoglobin content (tHb) was obtained. Changes in these signals were compared with variations in middle cerebral artery flow velocity 
obtained using transcranial Doppler. Cross clamping and later reperfusion of the external carotid artery did not affect any variable measured. After application of the internal carotid cross clamp, eight patients showed a fall in middle cerebral artery flow velocity. Of these, seven demonstrated a rapid and correlated fall in $\mathrm{HbO}_{2}$ signal, and a reciprocal rise in $\mathrm{Hb}$. The $\mathrm{CytO}_{2}$ and $\mathrm{tHb}$ remained unchanged. Recovery of the $\mathrm{HbO}_{2}$ and $\mathrm{Hb}$ concentrations towards preclamp baseline values occurred in three of these patients during the period of internal carotid cross clamping. The use of an intraoperative shunt $(n=3)$ was also accompanied by a recovery of middle cerebral artery flow velocity, $\mathrm{HbO}_{2}$, and $\mathrm{Hb}$, although preclamp baseline concentrations were not achieved during shunting. In those patients in whom complete spontaneous recovery had not occurred, release of the internal carotid cross clamp resulted in a rapid increase in $\mathrm{HbO}_{2}$ and decrease in $\mathrm{Hb}$ signal towards the respective baseline values. A hyperaemia evolved with significant increases in middle cerebral artery flow velocity and $\mathrm{HbO}_{2}$ to above baseline values $(\mathrm{p}<0 \cdot 05)$. tHb was also increased during the hyperaemic phase $(p<0.08)$. The potential use of near infrared spectroscopy in adults for monitoring cerebral hypoxic stress was discussed.

THE ROLE OF NITRIC OXIDE IN VASOSPASM AFTER SUBARACHNOID HAEMORRHAGE LD Watkins, TP Obrenovitch, L Symon. Department of Neurological Surgery, Institute of Neurology, London, UK

Evidence is accumulating that a basal production of the endothelium derived relaxing factor, nitric oxide (NO), plays a part in maintaining normal cerebrovascular tone. The purpose of this study was to examine whether scavenging of NO by haemoglobin after subarachnoid haemorrhage contributes to vasospasm. ${ }^{2}$ The effects of adventitial exposure to haemoglobin were studied by injecting test solutions into the cisterna magna of anaesthetised rats while using laser Doppler flowmetry to monitor the local cerebral blood flow in the striatum and at the cortical surface.

A dose dependent reduction in striatal blood flow was found in response to haemoglobin, equivalent to a maximal reduction in local cerebral blood flow of $40 \%$. This effect was comparable with that obtained with solutions of L-nitroarginine methyl ester, a specific blocker of nitric oxide synthase. Both effects could be antagonised by L-arginine, the natural substrate which is used to produce NO, but not by the stereoisomer D-arginine.

The findings confirm the hypothesis that adventitial exposure to free haemoglobin can produce reduction in local cerebral blood flow, and strongly suggest that, under the observed conditions, this effect is due to interference with the action of NO.

1 Moncada S, Palmer RMJ, Higgs EA. Pharmacol Rev 1991;43:109-42.

$2 \mathrm{Kim}$ P, Sundt TM Jr, Vanhoutte PM. f Neurosurg 1988;69:239-46.

\begin{tabular}{|c|c|c|c|c|c|c|c|c|}
\hline \multirow[b]{2}{*}{ Operation } & \multicolumn{5}{|c|}{ Glasgow outcome score } & \multirow{2}{*}{$\begin{array}{l}\text { Directly } \\
\text { home \% }\end{array}$} & \multirow{2}{*}{$\begin{array}{l}\text { Average } \\
\text { stay post-op } \\
\text { (days) }\end{array}$} & \multirow{2}{*}{$\begin{array}{l}\text { Average } \\
\text { follow up } \\
\text { (months) }\end{array}$} \\
\hline & 1 & 2 & 3 & 4 & 5 & & & \\
\hline $\begin{array}{l}\text { Standard } \\
\text { Burr hole }\end{array}$ & $\begin{array}{l}0 \% \\
2 \cdot 9 \%\end{array}$ & $\begin{array}{l}4 \% \\
0 \%\end{array}$ & $\begin{array}{l}0 \% \\
0 \%\end{array}$ & $\begin{array}{l}12.0 \% \\
11.8 \%\end{array}$ & $\begin{array}{l}84.0 \% \\
85 \cdot 3 \%\end{array}$ & $\begin{array}{l}52.0 \\
61.8\end{array}$ & $\begin{array}{l}9 \cdot 7 \\
8 \cdot 2\end{array}$ & $\begin{array}{r}10 \\
5\end{array}$ \\
\hline
\end{tabular}

MINIMALLY INVASIVE ANEURYSM SURGERY: IS IT SAFE?

RW Gullan, SP Harland, A Hussein. Neurosurgical Unit, Brook General Hospital, London, UK

A retrospective study comparing conventional pterional cranitomy with extended burr hole exposure for anterior circulation aneurysms is presented. Each group comprised consecutive cases. Only those with posterior circulation aneurysms or those requiring an interhemispheric approach were excluded. Pterional craniotomy was performed on 25 patients (age range 22-72, mean 48 years, 10 men, 15 women) for 28 aneurysms (single 20, multiple five). Extended burr hole was performed on 34 patients (age range 23-70, average 48 years, 11 men, 23 women) for 37 aneurysms (single 27, multiple seven). Preoperative WFNS grading was similar in both groups as was the average number of days from ictus to surgery (pterional craniotomy average $11 \cdot 0$, range $2-35$ days; burr hole average $11 \cdot 6$, range 1-39 days). Temporary clips were used in seven of the pterional craniotomy burr hole and three of the cases. Intraoperative rupture occurred and was controlled in three pterional craniotomy and five burr hole cases. Extension of the craniectomy to improve exposure was required in only two of the 34 burr hole operations. A similar quality of survival was seen in both groups (table). There were no intraoperative or early postoperative deaths (<one month) in either group. Likewise morbidity directly related to surgery was exceedingly low. One patient in the pterional craniotomy group deteriorated, however, to a persistent vegetative state (Glasgow outcome score 2) because of intense vasospasm. In the burr hole group a patient whose aneurysm could only be wrapped died from a massive rebleed at six weeks. Mild hemiparesis was seen at follow up in two patients from each series. Permanent weakness of the frontalis muscle occurred in three patients after pterional craniotomy and in one after burr hole. It is concluded from this series that it is safe to continue this technique. Details of the procedure were openly discussed.

THE ASSESSMENT OF CEREBRAL ARTERIAI AUTOREGULATION USING INDUCED OSCILLATIONS IN BLOOD PRESSURE

M Dirnhuber, A Birch, R Hartley-Davies, D Laycock, G Neil-Dwyer, DA Lang, F Ianotti, OCE Sparrow. Wessex Neurological Centre, Southampton General Hospital, Southampton, UK

Transcranial Doppler ultrasonography has demonstrated the onset of cerebral arterial autoregulation within five seconds of a change in cerebral perfusion pressure. ${ }^{12}$ If cerebral perfusion pressure is oscillating, the phase of the resulting oscillation in middle cerebral artery flow velocity (MCAV) will lead that of the CPP by an angle that is greater the faster the autoregulation

Fourteen volunteers performed 19 cycles of squatting for 10 seconds alternating with standing for 10 seconds, repeated during hyperventilation induced hypocapnoea and hypercapnoea. MCAV was recorded using $2 \mathrm{MHz}$ pulsed transcranial Doppler ultrasonography. Off line, the cycles were averaged and the phase differences between MCAV and blood pressure were derived by using Fourier analysis.

All subjects showed greater phase differences during normocapnoea than hypercapnoea ( $p<0.0002$, Wilcoxon signed rank test) and showed greater differences with hypocapnoea than with normocapnoea ( $p<$ 0.002).

As this method might form the basis of a quantitative test of cerebral autoregulation, 15 normocapnic (end tidal $\mathrm{CO}_{2} 32-38 \mathrm{~mm}$ $\mathrm{Hg}$ ) patients undergoing lumbar laminectomy were investigated, using intermittent positive pressure ventilation to induce the oscillation in blood pressure. Recordings were made for five minute periods. Mean phase differences were similar to the normocapnic group in the previous study, indicating that this method might be able to assess autoregulation intraoperatively.

1 Giller CA. Acta Neurochir (Wien) 1991;108.

2 Aaslid R, et al. Stroke 1989;20:45-52.

EFFECTS OF EARLIER TRANSFER, FLUID RESUSCITATION, AND SURGERY ON THE OUTCOME OF PATIENTS WITH ANEURYSMAI SUBARACHNOID HAEMORRHAGE

PC Whitfield, PJ Kirkpatrick, $\mathrm{H}$ Moss, D O'Hare. Department of Neurosurgery, Addenbrooke's Hospital, Cambridge, UK

The outcome in 214 consecutive patients presenting with subarachnoid haemorrhage to a regional neurosurgical unit was audited. During the study period a change in management protocol was introduced to encourage earlier admission and active fluid resuscitation. All patients received nimodipine. The timing of surgical intervention was assessed and the morbidity and mortality compared between different surgical groups. One hundred and thirty three $(62 \%)$ patients underwent aneurysm surgery. The mean day of surgery was reduced from day 16.9 in the first 75 patients to day 7.5 in the subsequent 58 patients. Seventy eight per cent of surgical patients had a good outcome (Glasgow outcome score $=1$ ) two months postoperatively. In 100 operative patients presenting with grade 1 or 2 subarachnoid haemorrhage, a Glasgow outcome score of 1 was achieved in $82 \%$ and was independent of the timing of surgery. In those with grades 3-5, a Glasgow outcome score of 1 was achieved in $67 \%$ and was also independent of the timing of surgery. The overall operative mortality was $5 \%$. Of the 81 nonoperated cases, 26 patients re-bled (median day 8.5$)$, leading to death in $23(88 \%) ; 39$ $(18 \%)$ cases had negative angiography.

With earlier transfer and resuscitation, 
surgery within six days of aneurysmal subarachnoid haemorrhage did not adversely affect surgical outcome. Earlier surgery reduces patient exposure to fatal rebleeds.

EFFECT OF GRADE OF SURGEON ON THE OUTCOME OF ANEURYSM SURGERY LM Myles, MG O'Sullivan, PFX Statham, IR Whittle, JD Miller, AJW Steers. Department of Clinical Neurosciences, Western General Hospital, Edinburgh, UK

The aim was to assess the effect of grade of surgeon on the intraoperative complications, the incidence of delayed ischaemic neurological deficit, and outcome of patients undergoing surgery for cerebral aneurysm.

A prospective study of all patients undergoing aneurysm surgery was carried out in this department in 1993. Patients were divided into two groups on the basis of their WFNS grade (good grade $=$ WFNS 1-3, poor grade $=$ WFNS 4 and 5) at the time of admission and immediately before surgery. The initial CT scan was graded with the method described by Hijdra et al. ${ }^{1}$ The site and size of the aneurysm was noted. Surgeons were graded according to their hospital grade and consultants were further subdivided into three groups according to time in post. The intraoperative incidence of aneurysm rupture, unsatisfactory clipping, or temporary clipping was recorded. Outcome was assessed at discharge or 30 days and at three months.

There were 63 operations for aneurysm32 by consultants, 26 by senior registrars and five by registrars (supervised by a consultant) in the study period. The age of the patients was not significantly different between the groups (mean $=48$ years). The preoperative WFNS grade was also similar between groups with about $70 \%$ of patients being in good grade immediately preoperatively and $30 \%$ poor grade. All posterior circulation aneurysms were operated on by one consultant surgeon. Delayed ischaemic deficits were noted in seven out of 63 patients $(11 \%)$ postoperatively. The incidence of intraoperative aneurysm rupture, temporary clipping, or unsatisfactory clip placement was not significantly different. Outcome at time of discharge was not related to grade of surgeon with $46 / 63$ patients having a good outcome $(73 \%)$.

In conclusion, grade of surgeon does not influence outcome from subarachnoid haemorrhage.

1 Hijdra A, et al. Stroke 1990;21:1156-61.

CRITICAL APPRAISAL OF DRAINAGE IN SYRINGOMYELIA

S Sgouros, B Williams. Midland Centre for Neurosurgery and Neurology, Smethwick, UK

The use of drains for syringomyelia has an immediate appeal and has been practised widely since the report of Abbe and Coley over 100 years ago. Good short term results have been claimed but long term outcome is largely unknown. An experience in Birmingham, United Kingdom, is reviewed. Eighty two patients who had some form of syrinx drainage procedure had 59 syringopleural and 15 syringosubarachnoid shunts. Ten years after their operation only $54.2 \%$ and $46.6 \%$ of the patients respectively remained stable. A $7 \%$ complication rate was recorded, including fatal haemorrhage and drain displacement. At least $5 \%$ of shunts were blocked at second operation or necropsy.

These data indicate that drainage procedures are not the answer to syringomyelia. The effect of other tubeless drainage procedures such as syringotomy and terminal ventriculostomy was also analysed and found not to offer substantial benefit. It is suggested that rather than attempt to drain the syrinx cavity by shunting its contents to a low pressure site, it is best to disable the filling mechanism of the syrinx. Most forms of syringomyelia have a blockage at the foramen magnum or in the subarachnoid space of the spine. Measures to reconstruct the continuity of the subarachnoid space at the blockage site are strongly recommended. Lowering of overall CSF pressure is advocated when re-establishment of the pathways proves impossible. Syrinx drainage as an adjuvant to elective surgery may continue to have a place.

RANDOMISED CONTROLLED TRIAL OF PERCUTANEOUS AND CONVENTIONAL TRACHEOSTOMY IN NEUROSURGICAI PATIENTS

C Bolger, R Gaffney, A Synnott, L Viani. Departments of Neurosurgery, ENT, Anaesthesia, Beaumont Hospital, Dublin, Eire

Percutaneous dilatational tracheostomy (PCT) was introduced by Ciaglia in 1985. The technique is quick and simple, being performed at the patient's bedside rather than in theatre. Published series and retrospective studies have suggested that PCT has a lower incidence of complications and is easier to perform than conventional tracheostomy.

This study prospectively compared PCT with conventional tracheostomy in relation to ease of performance, duration of procedure, and complication rate.

The study population consisted of neurosurgical patients requiring prolonged ventilation. All patients intubated for 10 days and unlikely to be extubated over the next four days were randomly assigned to receive PCT or conventional tracheostomy. Exclusion criteria included patients under the age of 18 and those with significant neck trauma. Informed consent was obtained from next of kin. For the purpose of this study all procedures were performed in theatre. To date 13 patients have had PCT and 11 patients conventional tracheostomy. PCT was successfully performed in all allocated patients. Early results indicate that PCT is quicker than conventional tracheostomy. One patient in the conventional group had a Staphylococcus aureus wound infection. Apart from this there were no complications. Late follow up is currently being undertaken and complications assessed. We conclude that PCT is a simple, cost effective, and safe alternative to conventional tracheostomy.

OSTEOPLASTIC $v$ FREE FLAP CRANIOTOMY: A PROSPECTIVE RANDOMISED TRIAI MS Eljamel, D Rawluk. Richmond Institute for Neurology and Neurosurgery, Beaumont Hospital, Dublin, Eire

Pterional craniotomy can be performed by either raising a skin muscle flap and remov- ing a bone flap (free flap craniotomy) or by leaving the bone flap attached to the temporalis muscle (osteoplastic flap). Free flap craniotomies have been associated with an increased infection rate, whereas osteoplastic craniotomies have been associated with injury to the temporal branches of the facial nerve and paralysis of the frontalis muscle. The aim of this study was to compare the two methods of craniotomy prospectively and directly.

Forty consecutive patients, who agreed to enter the trial, were randomised and operated on by the two authors. Twenty one patients had free flap craniotomies and 19 had osteoplastic flaps. The two groups were closely matched for age $(50.2(12.4) v 46.9$ (13.8) years), sex (8:13 $v 10: 9)$ and underlying pathology. Wounds were inspected daily for evidence of infection and frontalis muscle function was assessed at discharge and at three months. Analyses were by $\chi^{2}$ tests with Yates' correction and $p$ values were two tailed.

Five patients developed severe and two moderate frontalis muscle weakness, all of whom had osteoplastic flaps $(p<0.05)$ Two patients developed wound infections, both of whom had free flap craniotomies $(p>0.05)$. The mean duration of free flap craniotomy was $2 \cdot 1$ hours whereas osteoplastic flap took an average of 3.2 hours to perform $(p<0.01)$

In this trial, free flap craniotomy reduces injury to the frontalis muscle innervation, does not significantly increase infection rate, and is quicker to perform.

\section{SPINAL INTRADURAL TUMOURS: PRESTON} EXPERIENCE

S Perera, NT Gurusinghe. Department of Neurosurgery, Royal Preston Hospital, Preston, UK

It is well established that significant neurological deterioration occurs due to diagnostic delay in malignant extradural spinal cord compression. Although spinal intradural tumours are mostly benign slow growing lesions a good outcome is nevertheless determined by early diagnosis and prompt treatment. We have reviewed 142 consecutive cases over four decades (1954-93) which is the largest reported series in the United Kingdom in recent times and analysed the clinical features, the referral pattern, and diagnostic delay. The conclusions are as follows: (1) At the time of diagnosis only $23 \%$ of patients had normal gait, $35 \%$ had an abnormal gait, $23 \%$ walked with assistance, and $19 \%$ were bedridden. At that stage $58 \%$ of patients had clinical motor deficit and $30 \%$ of patients had impaired sphincter control. (2) Although $90 \%$ of patients presented to their general practitioner within six months of their symptoms, there was a delay of six months or more between the visit to the general practitioner and the first consultant referral in $35 \%$ and a similar delay in $35 \%$ between the first consultant and the neurosurgeon respectively. (3) Analysing the cause for the neurological deterioration showed that $90 \%$ of the patients were initially referred by the general practitioners to consultants in specialities other than Neurosurgery. (4) There was no significant change in the referral pattern and diagnostic delay during the pas decade despite the tremendous advances in the investigative techniques available. We think that this situation is of continuing concern. 